\title{
Long lasting mesalazine-induced pancytopenia in a patient with ulcerative colitis
}

\author{
Juyeon $\mathrm{Ko}^{1}$, Hyun Joo Song ${ }^{2, \mathbb{4}}$, Sanghoon Han ${ }^{2}$, Chiyoon Moon ${ }^{1}$ \\ ${ }^{1}$ Medical Course, Jeju National University School of Medicine \\ ${ }^{2}$ Department of Internal Medicine, Jeju National University School of Medicine
}

\begin{abstract}
Inflammatory bowel disease (IBD) is an immune-mediated chronic inflammatory intestinal condition. With development of various treatment options for IBD, 5-aminosalicylic acid (5-ASA) agents became the drugs of choice due to high efficacy and low risk of complication, specifically effective at inducing and maintaining remission in ulcerative colitis (UC). Pancytopenia caused by 5-ASA agents, especially by mesalazine, has been rarely reported compared with azathioprine, which is commonly used for glucocorticoid-dependent IBD and known to have risk of bone marrow suppression. In the present report, we describe the case of a 57-year-old woman diagnosed with UC, who developed pancytopenia due to adverse effect of mesalazine after recovery from azathioprine-induced pancytopenia. After withdrawal of mesalazine, the laboratory values consistent with myelosuppression continued for 3 months while pancytopenia from azathioprine remained only for 2 weeks. Since pancytopenia can be fatal due to its risk of severe bleeding and infection, close monitoring of clinical presentation is important when starting mesalazine and laboratory data should be evaluated whenever the patients present related symptoms. Furthermore, we suggest that complete blood cell counts should be considered when resuming mesalazine following the development of pancytopenia from any cause, as routinely recommended for azathioprine use.
\end{abstract}

Key words: Pancytopenia, 5-aminosalicylic acid, Mesalazine, Ulcerative colitis

\section{INTRODUCTION}

Commonly used drugs for treatment of mild to moderate inflammatory bowel disease (IBD) include 5-aminosalicylic acid (5-ASA) agents, which is specifically effective at inducing and maintaining long-term remission in ulcerative colitis (UC). ${ }^{1)}$

Despite therapeutic effects of 5-ASA agents, they also have a wide range of adverse effects. Common side effects include nausea, abdominal pain and headache, while uncommon hypersensitivity reactions including pancreatitis,

Received: August 6, 2019; Revised: August 26, 2019; Accepted: August 27, 2019 $\checkmark$ Correspondence to : Hyun Joo Song

Department of Internal Medicine, Jeju National University Hospital, Aran 13-gil 15, Jeju-Si, Jeju, 63241, Republic of Korea

Tel: 82-64-754-8142, FAX: 82-64-717-1131

E-mail: songhj@jejunu.ac.kr nephritis, pericarditis and paradoxical worsening of colitis have also been reported. ${ }^{2)}$ However, pancytopenia caused by 5-ASA was rarely reported compared with azathioprine, which is already known to have risk of bone marrow suppression (particularly leucopenia). ${ }^{3)}$

In the present report, we describe a case of pancytopenia in a 57-year-old woman with UC who was treated with mesalazine $\left(\right.$ Asacol $^{\circledR}$ ), a delayed-release sulfa-free 5-ASA preparation. This case differs from previous reports in that pancytopenia developed following recovery from 2 weeks of azathioprine-induced pancytopenia, with a much longer duration of 3 months.

\section{CASE REPORT}

A 57-year-old woman was diagnosed with ulcerative 

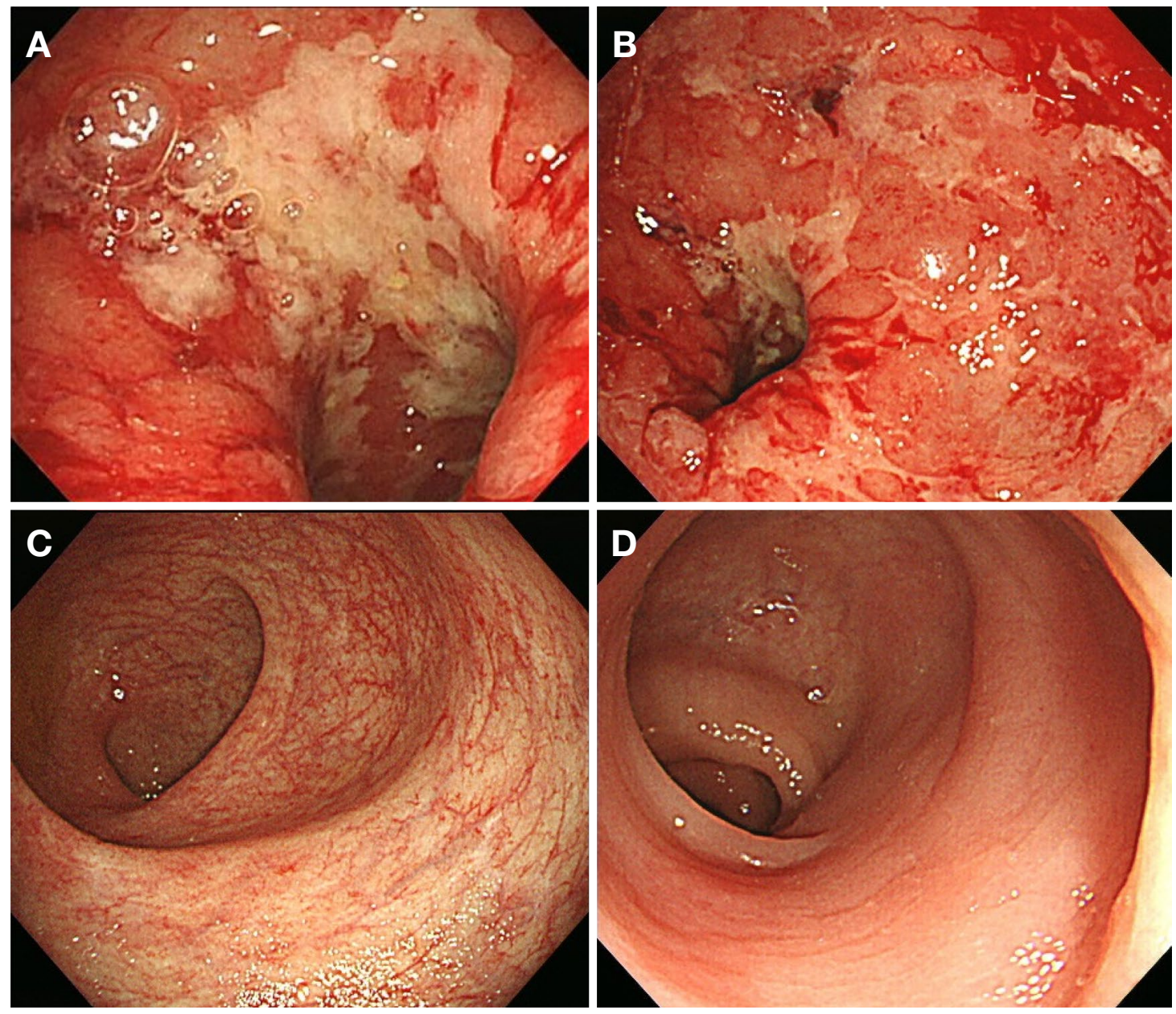

Figure 1. Colonoscopic findings of the patient with ulcerative colitis. (A, B) Colonoscopy showed severe, active ulcerations with hemorrhagic mucosa in the rectosigmoid area. (C, D) After discontinuation of azathioprine and mesalazine, complete remission was found 7 months later.

proctitis on February 19, 2014, based on her clinical presentation (diarrhea, hematochezia, abdominal pain and distension) and colonoscopy. The patient had started treatment of mesalazine $500 \mathrm{mg}$ /day as rectal suppository. After 8 months when the patient developed left-sided ulcerative colitis on colonoscopy, oral mesalazine $2.4 \mathrm{~g} /$ day was added with tapering dose of oral prednisolone from 40 to $5 \mathrm{mg} /$ week and suppository was changed into mesalazine enema $4 \mathrm{~g} / 100 \mathrm{~mL} /$ day.

Despite a 2-month course of mesalazine and prednisolone, her symptoms were aggravated due to severe disease activity (Fig. 1A, B); therefore, she was treated with azathioprine $50 \mathrm{mg}$ daily with decreased dose of mesalazine, $1.2 \mathrm{~g} / \mathrm{day}$. After 40 days use of azathioprine, she developed flu-like symptoms including fever and chill, and her complete blood cell counts showed pancytopenia (hemoglobin concentration, $7.3 \mathrm{~g} / \mathrm{dL}$; white blood cell count, $600 / \mathrm{mm}^{3}$ with absolute neutrophil count, $20 / \mathrm{mm}^{3}$; and platelet count, $18,000 / \mathrm{mm}^{3}$ ) that was assumed to be azathioprine-induced bone marrow suppression. She recovered 2 weeks later after discontinuing azathioprine, receiving recombinant G-CSF $75 \mu \mathrm{g} /$ day for 4 days and antibiotics (cefepime and metronidazole) for neutropenic fever.

Thereafter, the patient continued oral mesalazine $1.2 \mathrm{~g} /$ day without azathioprine due to active UC and was followed up for complete blood cell count, regularly visiting the hospital. Forty-five days after maintaining mesalazine only, she complained of petechiae on her neck, alopecia and aggravated hematochezia. Initial complete blood count on that day showed leucopenia $\left(1,500 / \mathrm{mm}^{3}\right)$ with absolute neutrophil count of $520 / \mathrm{mm}^{3}$. Hemoglobin concentration was $9.5 \mathrm{~g} / \mathrm{dL}$ with hematocrit of $27.0 \%$ and platelet count of $9,000 / \mathrm{mm}^{3}$. Within 3 days, the laboratory values be- 


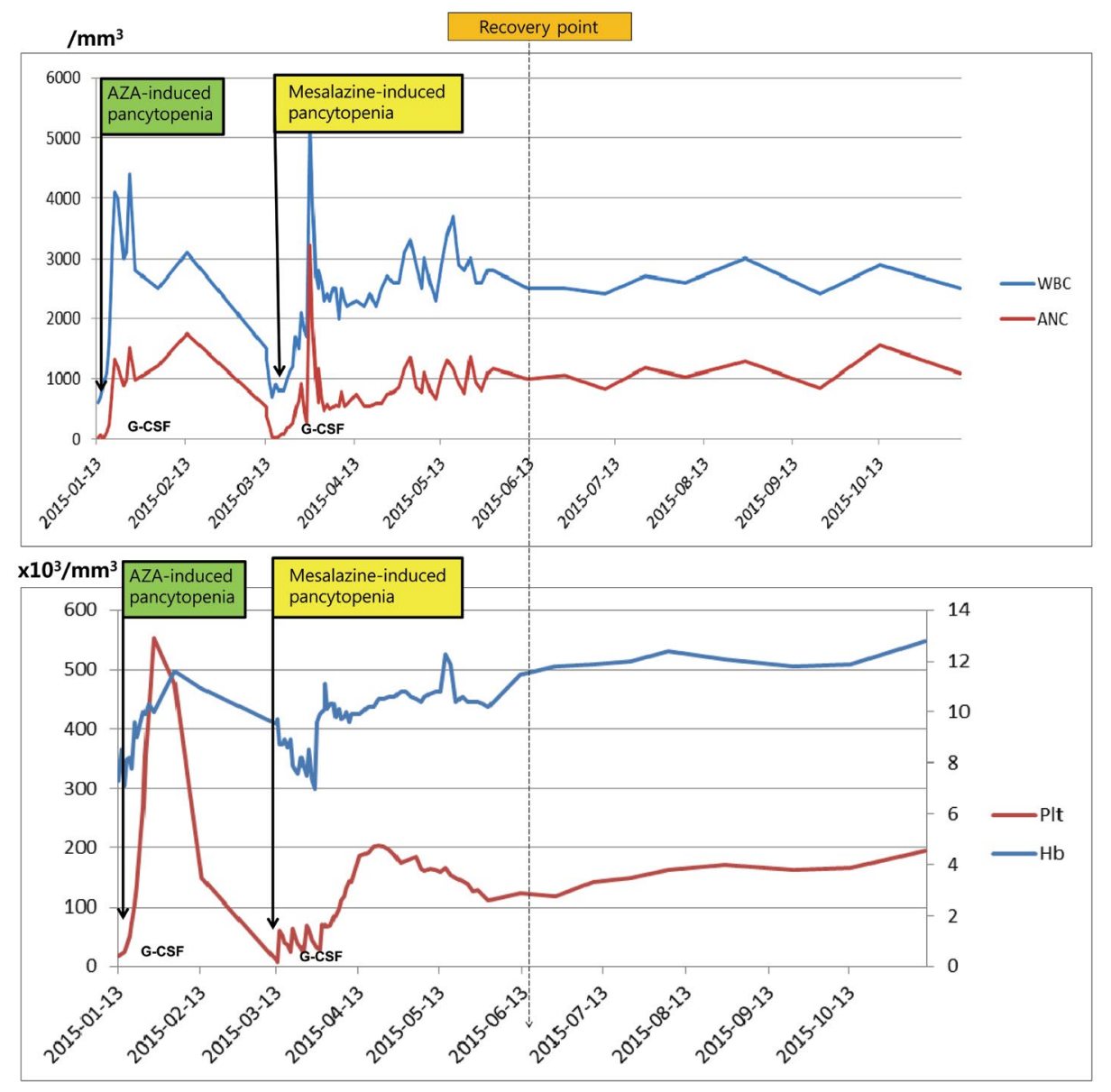

Figure 2. Changes of hematological indicators of the patient. WBC, white blood cell; ANC, absolute neutrophil count; Plt, platelet; Hb, hemoglobin.

came aggravated; white blood cell count was $800 / \mathrm{mm}^{3}$; absolute neutrophil count $20 / \mathrm{mm}^{3}$; hemoglobin concentration $8.6 \mathrm{~g} / \mathrm{dL}$; and platelet count $24,000 / \mathrm{mm}^{3}$.

Reticulocyte rate was $0.08 \%$ (normal: $0.5 \sim 1.5 \%$ ), with serum iron of $111 \mu \mathrm{g} / \mathrm{dL}$ (normal: $43 \sim 172 \mu \mathrm{g} / \mathrm{dL}$ ), ferritin of $612 \mu \mathrm{g} / \mathrm{dL}$ (normal: $13 \sim 150 \mu \mathrm{g} / \mathrm{dL}$ ) and total iron binding capacity (TIBC) of $235 \mu \mathrm{g} / \mathrm{dL}$ (normal: 196 567 $\mu \mathrm{g} / \mathrm{dL}$ ). Folate or vitamin B12 deficiency was absent. Peripheral blood smear showed pancytopenia; red blood cell was normocytic normochromic; white blood cell count moderately decreased in number with relative lymphocytosis neutropenia; platelet count also markedly decreased in number without clumping. Erythrocyte sedimentation rate was $10 \mathrm{~mm} / \mathrm{hr}$ (normal: $0 \sim 20 \mathrm{~mm} / \mathrm{hr}$ ) and C-reactive protein was $0.71 \mathrm{mg} / \mathrm{dL}$ (normal: $0.00 \sim 0.30 \mathrm{mg} / \mathrm{dL}$ ).

Other laboratory data, including liver and renal function, as well as serology for viral or bacterial infection and for auto-antibodies $(\mathrm{Ab})$ of rheumatic disease, were normal. Additional data included: aspartate aminotranferase, 17 IU/
L; alanine aminotransferase, 21 IU/L; alkaline phosphatase, $204 \mathrm{U} / \mathrm{L}$; blood urea nitrogen, $14.6 \mathrm{mg} / \mathrm{dL}$; creatinine, $0.9 \mathrm{mg} / \mathrm{dL}$; culture, polymerase chain reaction, antigen or antibody detection for Cytomegalovirus, Influenza virus, Hepatitis B, C virus, Human immunodeficiency virus, $M y$ cobacterium, Streptococcus pneumoniae, Legionella and Mycoplasma, all negative; anti-cardiolipin $\mathrm{Ab}$, anti-platelet $\mathrm{Ab}$, anti-SSa/Ro Ab, anti-SSb/La Ab, anti- $\beta 2$ glycoprotein I (GPI) Ab, anti-neutrophil cytoplasmic antibody (ANCA), rheumatoid factor (RF), all negative.

During admission, the patient immediately discontinued mesalazine and hemato-oncologist was consulted to evaluate the cause and course of pancytopenia. The patient was treated with recombinant G-CSF injection $300 \mu \mathrm{g} /$ day until absolute neutrophil count recovered greater than 500/ $\mathrm{mm}^{3}$, with platelet transfusion whenever platelet count fell below $30,000 / \mathrm{mm}^{3}$ and antibiotics (cefepime). Complete blood cell counts were closely monitored every other day.

After withdrawal of mesalazine, pancytopenia contin- 
ued for 3 months. In comparison with hemoglobin and platelet count which showed a relatively stable course of recovery, the absolute neutrophil count very slowly recovered with fluctuation (Fig. 2). Bone marrow biopsy was recommended on several occasions to evaluate primary bone marrow disease due to the fluctuating course of neutropenia, but the patient refused and the medical team decided to continue follow-up, since the hematologic data was slowly improving.

After 3 months, the patient was considered to be in recovery as the laboratory profiles became stable; complete remission was achieved 7 months after discontinuation of azathioprine and mesalazine (Fig. 1C, D). After 2 years of remission the disease returned as evaluated on sigmoidoscopy, the patient was treated with oral prednisolone on a tapering dose of 30 to $5 \mathrm{mg} /$ day for 2 months since she could not use both azathioprine and mesalazine. There was no noticeable problem with the treatment and thereafter, and the patient has been regularly evaluated without symptom recurrence during 4 years.

\section{DISCUSSION}

The development of a wide range of treatment options including 5-ASA agents, glucocorticoids, immunosuppressant drugs and biologic agents have made it possible to induce and maintain remission of IBD, improving the quality of life of IBD patients and even reducing the likelihood of intestinal surgery. ${ }^{4)}$ However, beyond the therapeutic effects it is also important to recognize the toxicity of the drugs according to their pharmacologic characteristics.

Azathioprine is a purine analogue in which metabolites inhibit purine ribonucleotide synthesis, cell proliferation and immune response. Bone marrow suppression with leucopenia is a well-known risk of azathioprine ${ }^{5)}$ and the relationship with individual thiopurine s-methyltransferase (TPMT) allele has been studied recently. ${ }^{6}$ According to the study by Konidari et al., ${ }^{7}$ reported incidence of myelotoxicity and leucopenia due to thiopurine adverse effects were $4 \%$ and 2 to $15 \%$, respectively. Since bone marrow suppression is dose-related and even delayed ${ }^{2)}$, it routinely necessitates regular monitoring of complete blood cell counts.

However, 5-ASA agents, which consist of 5-aminosalicylic acid, are known to be tolerable compared with azathioprine and other immunomodulators. There are several types of 5-ASA agents that can be classified roughly into sulfasalazine and sulfa-free preparations.

Sulfasalazine was originally developed to deliver both antibacterial (sulfapyridine) and anti-inflammatory effects into connective tissues of colonic mucosa. Up to a third of patients treated with sulfasalazine have reported side effects, which are thought to be related to the sulfur part of the molecule. ${ }^{8)}$ Common adverse effects include headache, nausea, anorexia and malaise while other allergic or toxic adverse effects include fever, rash, hemolytic anemia, hepatitis, pancreatitis, paradoxical worsening of colitis, and reversible sperm abnormalities. ${ }^{2)}$

The newer category, sulfa-free aminosalicylate formulations, include alternative azo-bonded carriers (Olsalazine, Balsalzine), delayed-release (Delzicol, Asacol HD, Lialda), controlled-release (Pentasa), extended-release (Apriso) preparations, the latter three of which are known as mesalazine or mesalamine-based oral 5-ASA agents. Though they are not proven to be superior to sulfasalazine in efficacy ${ }^{8)}$, their adverse effects are less frequent as they were developed to avoid sulfur-related reaction. Nausea, dyspepsia, headache and uncommon hypersensitivity reactions such as worsening of colitis, pancreatitis, pericarditis, and nephritis are reported. ${ }^{2)}$

Though rare, 5-ASA agents can cause severe hematologic complications that are potentially fatal. Sulfasalazine has been reported to be associated with bone marrow suppression such as leucopenia, agranulocytosis, thrombocytopenia, pancytopenia, hemophagocytosis, erythroid hypoplasia and bone marrow necrosis, ${ }^{5,9)}$ with these side effects developing more frequently than from mesalazine. According to a United Kingdom population-based study by Jick et al. ${ }^{10)}$, the frequency of blood disorders from sulfasalazine was $27 / 10,332$ (2.6/1000 user), compared with zero case of blood disorders in users of mesalazine. Though relatively rare, bone marrow suppression from mesalazine has also been reported, as well as 5 cases of pancytopenia. $^{11-15)}$ (Table 1)

We reported here a case of pancytopenia from mesalazine which differs from previous reports in that it developed after recovery from azathioprine-induced pancytopenia. At the time of presenting complication, the patient has resumed mesalazine $1.2 \mathrm{~g}$ /day for 45 days after discontinuing 40-day-use of azathioprine $50 \mathrm{mg} / \mathrm{day}$, and in total had taken mesalazine for 13 months (systemic use for 5 months) since she was first diagnosed with UC.

Of importance, two highlights of the case are here; first, the patient developed pancytopenia consistent with bone marrow suppression due to mesalazine, which is rarely reported to be associated with blood disorder; Second, the 
Juyeon Ko, Hyun Joo Song, Sanghoon Han, Chiyoon Moon

Table 1. Five case reports of mesalazine-induced pancytopenia and the present case

\begin{tabular}{|c|c|c|c|c|c|}
\hline References & Sex & Age & Type of IBD & Drug dose & Duration of drug \\
\hline Abboudi, ${ }^{11} 1994$ & M & 71 & $\mathrm{UC}$ & $1.6 \mathrm{~g} /$ day & 4 months \\
\hline Laidlow, ${ }^{12} 1994$ & M & 48 & $\mathrm{UC}$ & $1.2 \mathrm{~g} / \mathrm{day}$ & 12 months \\
\hline Otsubo, ${ }^{13} 1998$ & M & 20 & $\mathrm{UC}$ & $1.0 \mathrm{~g} /$ day & 5 months \\
\hline Kotanagi, ${ }^{14} 1998$ & M & 29 & $\mathrm{CD}$ & $3.0 \mathrm{~g} /$ day & 2 days \\
\hline Wiesen, ${ }^{15} 2009$ & $\mathrm{~F}$ & 52 & $\mathrm{CD}$ & $3.0 \mathrm{~g} /$ day & several years \\
\hline \multirow[t]{3}{*}{ Present case } & $\mathrm{F}$ & 57 & $\mathrm{UC}$ & $2.4 \mathrm{~g} /$ day & 2 months \\
\hline & & & & $1.2 \mathrm{~g} / \mathrm{day}$ & 3 months \\
\hline & & & & (after 8 months of suppository) & \\
\hline
\end{tabular}

CD, Crohn's disease; UC, ulcerative colitis

course of pancytopenia from mesalazine use remained much longer at 3 months compared to 2 weeks of azathioprine-induced pancytopenia.

This case demonstrates the importance of careful monitoring of clinical presentations and, if needed, laboratory evaluation when using mesalazine. This is especially relevant when mesalazine is resumed after recovery from hematologic complication from any cause (in this case, from azathioprine). Clinical presentations of pancytopenia range variously from petechiae, purpura, easy bruising precipitated by thrombocytopenia, flu-like symptoms such as fever and chill due to infection precipitated by neutropenia to even non-specific forms, so evaluation of symptoms related to cytopenia should be done.

Limitations of this study include: first, bone marrow biopsy was not examined, so that primary bone marrow disease could not be ruled out. However, it must be considered that the laboratory values remained stable within normal range for 11 months from February 19, 2014 (when diagnosed with UC) to January 13, 2015 (when the patient first developed pancytopenia from azathioprine), and that the patient developed pancytopenia abruptly when she resumed mesalazine only; this indicates a strong association with secondary bone marrow depression due to adverse effect of mesalazine, whether it was concurrent with the primary marrow disease or not.

Second, since the patient developed first pancytopenia from azathioprine and was stable when she had used mesalazine for 2 months previously, it is possible to assume that the second event also resulted from azathioprine toxicity, considering its variation at the time needed between presentation and recovery from myelosuppression. ${ }^{3,16)}$ However, if it is from prolonged drug toxicity, the laboratory values including absolute neutrophil counts generally remained low, considering that they were normalized and remained stable for a few weeks without G-CSF maintenance in this case. Also, there is no case reported in which azathioprine induced a second event of myelosuppression during which hematologic profiles become normalized.

The clear mechanism that explains mesalazine-induced hematologic complication has not been established and the risk might be underestimated compared with azathioprine. However, a few cases of bone marrow suppression from mesalazine use have been reported and this case also supports the previous studies. Therefore, we recommend immediate discontinuation of the drug whenever patients present signs and symptoms of pancytopenia. Useful management may include G-CSF injection and prophylactic antibiotics. Furthermore, our findings strongly suggest that regular and close monitoring of laboratory profiles such as complete blood cell counts should be considered when resuming mesalazine after having developed pancytopenia, as routinely recommended for azathioprine use.

\section{CONFLICT OF INTEREST}

No potential conflict of interest relevant to this article was reported.

\section{AUTHOR CONTRIBUTION}

Song HJ designed the report; Ko J collected the clinical data and wrote the paper; Han S and Moon C analyzed the data and edited.

\section{REFERENCES}

1. Hauso Ø, Martinsen TC, Waldum H. 5-aminosalicylic acid, a specific drug for ulcerative colitis. Scand J Gastroenterol 2015;50:93341.

2. Stein RB, Hanauer SB. Comparative tolerability of treatments for 
inflammatory bowel disease. Drug Saf 2000;23:429-48.

3. Connell WR, Kamm MA, Ritchie JK, Lennard-Jones JE. Bone marrow toxicity caused by azathioprine in inflammatory bowel disease: 27 years of experience. Gut 1993;34:1081-5.

4. Chatu S, Saxena S, Subramanian V, Curcin V, Yadegarfar G, Gunn $\mathrm{L}$, et al. The impact of timing and duration of thiopurine treatment on first intestinal resection in crohn's disease: national UK population-based study 1989-2010. Am J Gastroenterol 2014;109:409-16.

5. Cunliffe RN, Scott BB. Review article: monitoring for drug side-effects in inflammatory bowel disease. Aliment Pharmacol Ther 2002;16:647-62.

6. Cabaleiro T, Roman M, Gisbert JP, Abad-Santos F. Utility of assessing thiopurine s-methyltransferase polymorphisms before azathioprine therapy. Curr Drug Metab 2012;13:1277-93.

7. Konidari A, Matary WE. Use of thiopurines in inflammatory bowel disease: safety issues. World J Gastrointest Pharmacol Ther 2014;5:63-76.

8. Wang Y, Parker CE, Bhanji T, Feagan BG, MacDonald JK. Oral 5-aminosalicylic acid for induction of remission in ulcerative colitis. Cochrane Database Syst Rev 2016;4:CD000543.

9. Abdulrahman SA. Sulfasalazine-induced pancytopenia indic- ative of bone marrow suppression: a case report. J Med Cases 2014;5:289-91.

10. Jick H, Myers MW, Dean AD. The risk of sulfasalazine- and mesalazine-associated blood disorders. Pharmacotherapy 1995;15:17681.

11. Abboudi ZH, Marsh JC, Smith-Laing G, Gordon-Smith EC. Fatal aplastic anaemia after mesalazine. Lancet 1994;343:542.

12. Laidlaw ST, Reilly JT. Antilymphocyte globulin for mesalazine-associated aplastic anaemia. Lancet 1994;343:981-2.

13. Otsubo H, Kaito K, Sekita T, Shimada T, Kobayashi M, Hosoya T. Mesalazine-associated severe aplastic anemia successfully treated with antithymocyte globulin, cyclosporine and granulocyte colony-stimulating factor. Int J Hematol 1998;68:445-8.

14. Kotanagi H, Ito M, Koyama K, Chiba M. Pancytopenia associated with 5-aminosalicylic acid use in a patient with Crohn's disease. J Gastroenterol 1998;33:571-4.

15. Wiesen A, Wiesen J, Limaye S, Kaushik N. Mesalazine-induced aplastic anemia. Am J Gastroenterol 2009;104:1063.

16. Gisbert JP, Gomollón F. Thiopurine-induced myelotoxicity in patients with inflammatory bowel disease: a review. Am J Gastroenterol 2008;103:1783-800. 